\title{
Detection of Redox Imbalance in Normal Lymphocytes with Induced Mitochondrial Dysfunction - EPR Study
}

\author{
EKATERINA GEORGIEVA ${ }^{1}$, ZHIVKO ZHELEV ${ }^{1,2}$, ICHIO AOKI ${ }^{3}$, \\ RUMIANA BAKALOVA ${ }^{3,4}$ and TATSUYA HIGASHI ${ }^{3}$ \\ ${ }^{1}$ Medical Faculty, Trakia University, Stara Zagora, Bulgaria; \\ ${ }^{2}$ Institute of Biophysics \& Biomedical Engineering, Bulgarian Academy of Sciences, Sofia, Bulgaria; \\ ${ }^{3}$ Department of Molecular Imaging and Theranostics, National Institute of Radiological Sciences, \\ National Institute for Quantum and Radiological Science and Technology, Chiba, Japan; \\ ${ }^{4}$ Medical Faculty, Sofia University St. Kliment Ohridski, Sofia, Bulgaria
}

\begin{abstract}
The present study describes a new approach for direct imaging of redox status in live cells using paramagnetic spin-probes, which allows evaluation of the level of oxidative stress due to overproduction of superoxide. The method is based on redox cycling of cell/mitochondriapenetrating nitroxide radicals (e.g. mito-TEMPO) and their electron-paramagnetic resonance (EPR) contrast, which makes them useful molecular sensors for analysis of redox status and oxidative stress in cells and tissues. Oxidative stress was induced in normal human lymphocytes by treatment with 2-methoxyestradiol and rotenone (ME/Rot) at different concentrations. This combination provokes mitochondrial dysfunction, which is accompanied by overproduction of superoxide. The EPR measurements were performed in dynamics on X-Band spectrometer after addition of mito-TEMPO to cell suspensions. The intensity of the EPR signal in untreated cells decreased significantly, which indicates a conversion of paramagnetic mito-TEMPO to its non-contrast diamagnetic form (hydroxylamine - mitoTEMPOH) due to reduction. In ME/Rot-treated cells, the signal decreased more slowly and to a lower level with increasing the concentration of ME/Rot. These data indicate an induction of oxidative stress in the cells in a concentration-dependent manner. A very good positive correlation between the intensity of EPR signal of mito$T E M P O$ and the intracellular level of superoxide was found,
\end{abstract}

Correspondence to: Rumiana Bakalova, Ph.D., D.Sci., Department of Molecular Imaging and Theranostics, National Institute of Radiological Sciences (NIRS), National Institute for Quantum and Radiological Science and Technology (QST), 4-9-1 Anagawa, Inageku, Chiba 263-8555, Japan. E-mail: bakalova.rumiana@qst.go.jp

Key Words: Nitroxides, superoxide, electron paramagnetic resonance imaging, mitochondrial dysfunction. analyzed by conventional dihydroethidium test $(R=0.9143$, $p<0.001)$. In conclusion, our study demonstrated that cellpenetrating paramagnetic spin-probes, such as mito$T E M P O$, are valuable tools for EPR imaging of the superoxide level in live cells, as well as for EPR imaging of mitochondrial dysfunction and metabolic activity, accompanied by superoxide imbalance.

Over 50 years of experience in free-radical biology and medicine has shown the crucial role of redox signaling in carcinogenesis, neurodegeneration, inflammation, and other pathologies (1-3). The cells and tissues of healthy mammals are characterized by low steady-state levels of oxidizers (e.g. reactive oxygen species, ROS) and higher steady-state levels of reducers (e.g. endogenous redox pairs: NADH/NAD ${ }^{+}$, $\mathrm{NADPH} / \mathrm{NADP}^{+}, \mathrm{FADH}_{2} / \mathrm{FAD}$, reduced/oxidized glutathione, reduced/oxidized ascorbate, etc. .). It is widely accepted that increasing ROS above a critical level provokes oxidative stress, which results in redox imbalance and triggers pathogenesis (1-3).

Currently, there is no universal methodology for the estimation of the cellular redox status in living cells. The oxidizing and reducing capacity of cells is determined by many parameters (e.g. ROS of different types and origin, products of free radical oxidation of biomacromolecules, status of natural non-enzymatic and enzymatic antioxidant systems, status of endogenous redox pairs, etc.) (1-5). Each parameter is analyzed separately by different methodologies. The estimation of cellular redox status is based on comparative analysis of one or several of these parameters and the conclusions are usually controversial.

The present study describes a new approach for direct imaging of redox status in isolated live cells, which allows evaluation of the level of oxidative stress due to overproduction of superoxide. The method is based on the redox cycle of cell-penetrating aminoxyl (nitroxide) spin- 


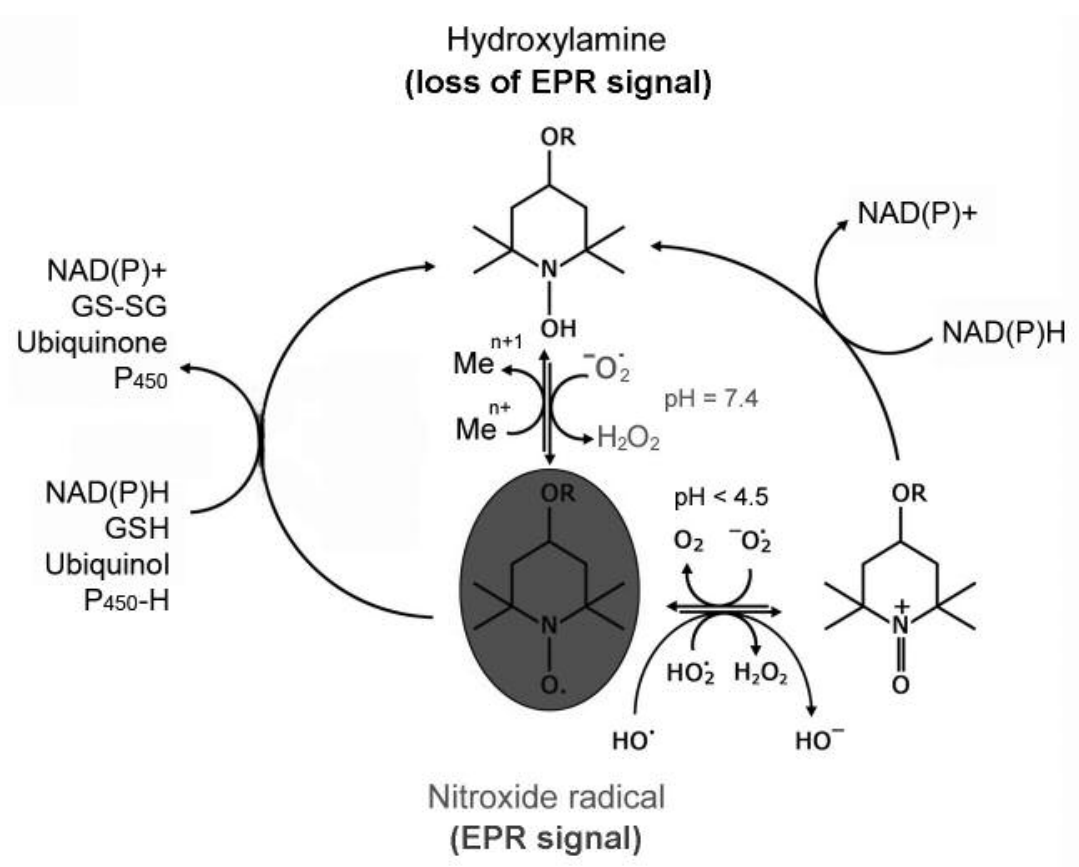

Figure 1. Redox-cycle of nitroxide spin-probes as a sensing platform for imaging of cellular redox status: principle of the method [according to Zhelev et al. (6)]. EPR: Electron-paramagnetic resonance.

probes and their electron-paramagnetic resonance (EPR) contrast, which makes them useful molecular sensors for visualization and evaluation of cellular redox balance $(6,7)$. The paramagnetic nitroxide radicals participate in electrontransfer reactions with oxidizers and reducers, with formation of non-contrast diamagnetic intermediate products (Figure 1). The rate constants of these reactions determine the intensity and dynamics of nitroxide-enhanced EPR signal in living cells.

In vitro studies demonstrated that the nitroxide radical can be converted rapidly to non-contrast diamagnetic forms, hydroxylamine and oxoammonium, by different cellular compounds (free ions of transition metals, superoxide and hydroperoxyl radicals, ubiquinols, $\mathrm{NAD}(\mathrm{P}) \mathrm{H}$, glutathione, ascorbate, etc.) (8-14). Hydroxylamine and oxoammonium are superoxide dismutase (SOD) mimetics and can restore nitroxide radical $(10,12,15,16)$. The interaction of oxoammonium with superoxide occurs very fast (rate constant $\sim 10^{9} \mathrm{M}^{-1} \mathrm{~s}^{-1}$ ) (16). However, under physiological conditions, oxoammonium can be reduced effectively to hydroxylamine by $\mathrm{NAD}(\mathrm{P}) \mathrm{H}$ (12). Hydroxylamine also interacts effectively with superoxide at physiological $\mathrm{pH} \sim 7.4$ with recovery of the contrast form (16-18). Therefore, in living cells and tissues, various reducers and oxidizers can be involved (directly or indirectly) in the formation of oxoammonium and hydroxylamine, but only the interaction of hydroxylamine with superoxide can restore the radical form of nitroxide and its EPR contrast. Thus, the dynamics of EPR signals of cell-penetrating nitroxide radicals might serve as a marker of oxidative stress, induced by overproduction of superoxide.

In order to test this assumption, we used an experimental model described by Huang et al. (19) and Pelicano et al. (20). The authors used rotenone, an inhibitor of mitochondrial complex-I, in combination with 2methoxyestradiol (ME), an inhibitor of mitochondrial Mndependent SOD, to test their effect on cellular superoxide levels by flow cytometry (Figure 2). They found that the combination of ME with rotenone (Me/Rot) caused an accumulation of high amounts of superoxide in cells and strongly minimized the production of hydrogen peroxide.

We applied this experimental strategy to cause overproduction of superoxide and to induce oxidative stress in normal human lymphocytes, as well as to clarify its effect on the dynamics of EPR signals of the nitroxide radical (2(2,2,6,6-tetramethylpiperidin-1-oxyl-4-ylamino)-2oxoethyl)triphenylphosphonium chloride (mito-TEMPO) in normal human lymphocytes.

\section{Materials and Methods}

Reagents. Mito-TEMPO was purchased from Enzo Life Sciences, Exeter, UK. All chemicals were analytical or HPLC grade. Deionized water (Milli-Q) was used in all model systems. 


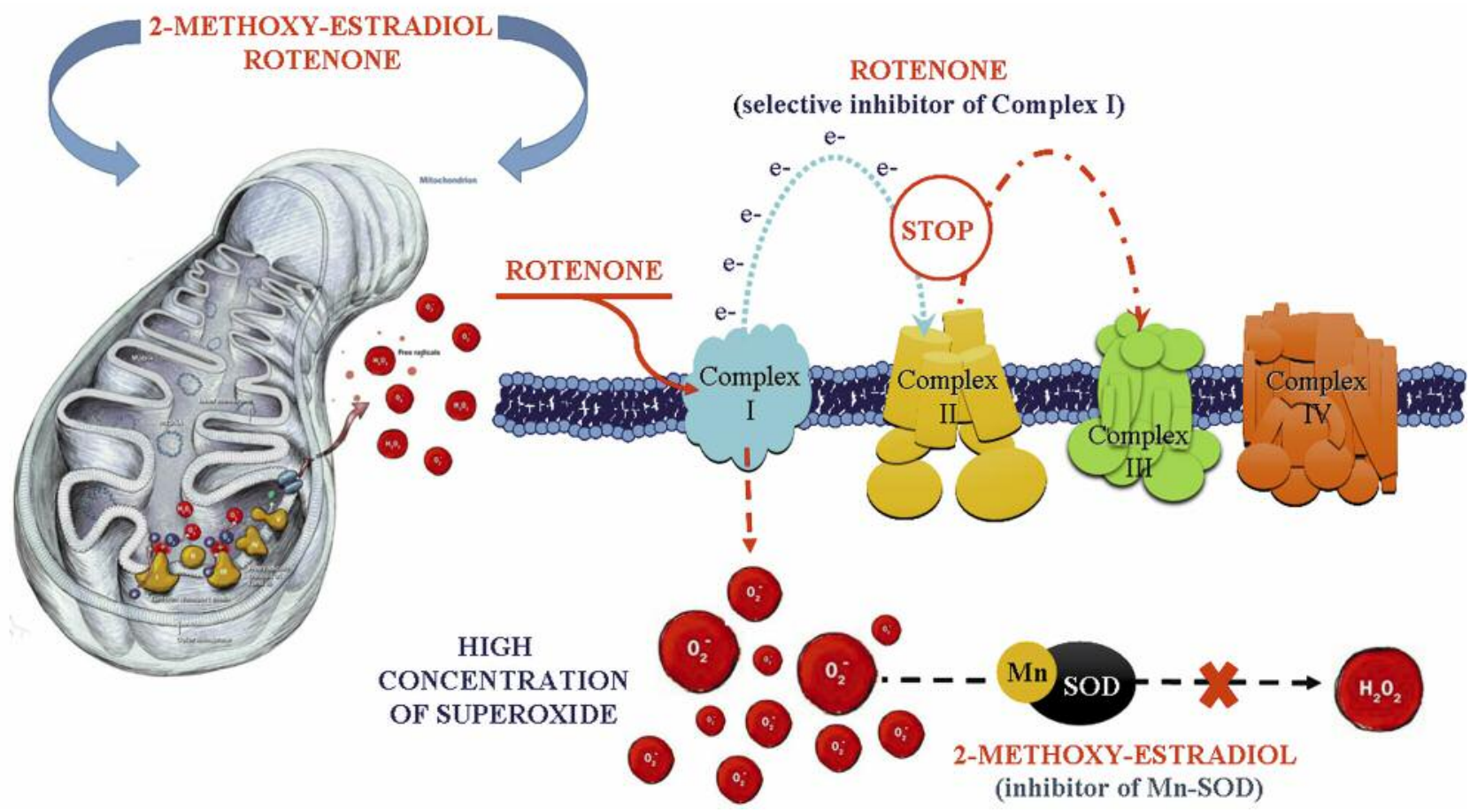

Figure 2. Biochemical strategy for enhancing superoxide accumulation in cells by inhibition of mitochondrial electron transport chain and mitochondrial superoxide dismutase (Mn-SOD) [according to Huang et al. (19) and Pelicano et al. (20)].

Cells. Normal human lymphocytes were isolated from heparinized blood of healthy adults (aged 38-40 years) using Lymphosepar-I (Immuno-Biological Laboratories, Takasaki, Japan). The cells were washed five times with phosphate-buffered saline (PBS; $10 \mathrm{mM}, \mathrm{pH}$ 7.4) and twice with RPMI-1640 medium, to eliminate traces of transition metals, which can compromise the results. Finally, the cells were re-suspended in the same culture medium and kept in a humidified atmosphere $\left(37^{\circ} \mathrm{C}, 5 \% \quad \mathrm{CO}_{2}\right)$ for 2 weeks. All experiments were performed within this time interval.

Cell treatment. Twenty-four hours before the experiment, the cells were re-suspended in fresh RPMI-1640 medium. Cell suspension $\left(1.0 \times 10^{6}\right.$ cells $\left./ \mathrm{ml}\right)$ was divided into two parts: non-treated and treated with ME/Rot. Each cell suspension was incubated for $12 \mathrm{~h}$ in a cell incubator.

Two different protocols were performed to induce mitochondrial dysfunction: (i) cells were pre-incubated in the absence or presence of $\mathrm{ME} / \mathrm{Rot}$ (base concentration $=300 \mathrm{nM} \mathrm{ME}$ and $250 \mathrm{nM}$ rotenone) and then mito-TEMPO $(0.1 \mathrm{mM})$ was added to the cell suspensions; (ii) cells were pre-incubated in the absence or presence of ME/Rot at different concentrations (2-, 5-, 10-, 20-fold the base concentration) and then mito-TEMPO $(0.1 \mathrm{mM})$ was added to the cell suspensions. The incubation was continued at $37^{\circ} \mathrm{C}$ in a humidified atmosphere. Aliquots of each cell suspension were collected at different time intervals and EPR measurements were performed at room temperature $\left(20-23^{\circ} \mathrm{C}\right)$.

Before each EPR experiment, the cells were counted and their viability was established by using trypan blue staining and Countess $^{\mathrm{TM}}$ automated cell counter (Invitrogen, OR, USA). The cell viability was in the range $92-95 \%$.
EPR measurements. EPR experiments were performed on an X-Band spectrometer (JEOL Inc., Peabody, MA, USA) with standard cavity, at the following parameters: microwave frequency: $9.4 \mathrm{GHz}$; field strength $=336 \mathrm{mT}$; field modulation frequency $=100 \mathrm{kHz}$; field modulation amplitude $=0.063 \mathrm{mT}$; microwave power: $2.0 \mathrm{~mW}$; time constant $=0.01 \mathrm{~s}$; sweep width $=10 \mathrm{mT}$; scan time (sweep time) $=1 \mathrm{~min}$, a number of scans: 1 . The EPR spectra were integrated and the data were calculated as a percentage from that of the control $(0.1 \mathrm{mM}$ of Mito-TEMPO, dissolved in cell-free medium).

Dihydroethidium (DHE) assay. DHE is a cell-penetrating fluorogenic probe, interacting predominantly with superoxide. The DHE assay allows distinguishing between superoxide and hydrogen peroxide $(21,22)$.

Briefly, DHE was dissolved in dimethyl sulfoxide to $65 \mathrm{mM}$ stock solution (kept at $-40^{\circ} \mathrm{C}$ ), which was diluted with PBS to prepare $50 \mu \mathrm{M}$ DHE working solution on the day of experiment. Ten microlitres of $50 \mu \mathrm{M}$ DHA were added to $100 \mu \mathrm{l}$ of each cell suspension. The samples were incubated for $15 \mathrm{~min}$ at room temperature and washed three times with PBS. The fluorescence intensity was detected immediately at $\lambda_{\mathrm{ex}}=518 \mathrm{~nm}$ and $\lambda_{\mathrm{em}}=605 \mathrm{~nm}$, using a microplate reader (TECAN Infinite ${ }^{\circledR}$ M1000; Tecan Austria $\mathrm{GmbH}$, Grodig, Austria).

\section{Results and Discussion}

The data in Figure 3 demonstrate the dynamics of EPR signals of mito-TEMPO in untreated and ME/Rot-treated (base concentration) normal human lymphocytes. In 


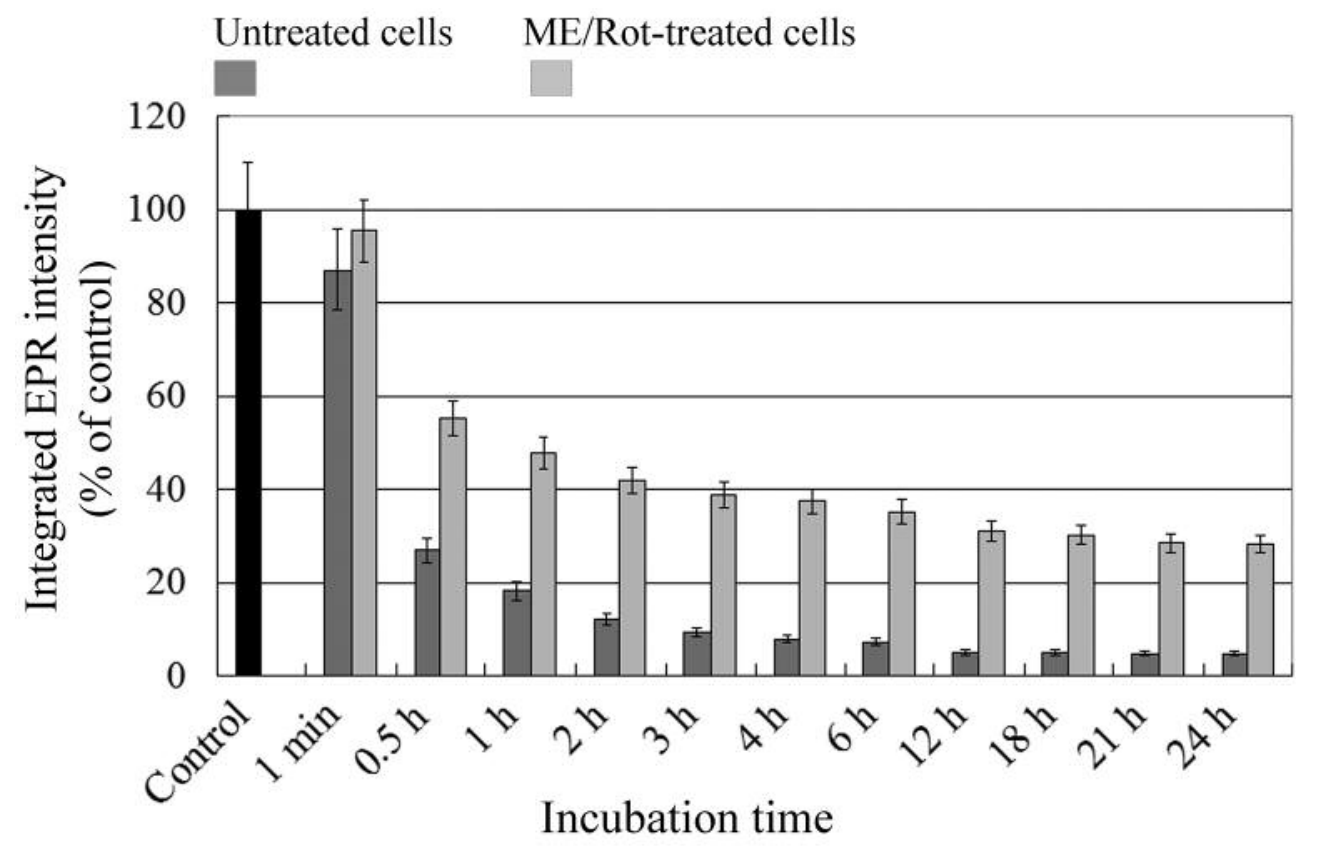

Figure 3. Dynamics of electron-paramagnetic resonance (EPR) signal of (2-(2,2,6,6-tetramethylpiperidin-1-oxyl-4-ylamino)-2oxoethyl)triphenylphosphonium chloride (mito-TEMPO) in untreated and 2-methoxy-estradiol (ME)/rotenone (Rot)-treated normal human lymphocytes. Experimental conditions: Cells $\left(1 \times 10^{6} \mathrm{cells} / \mathrm{ml}\right)$ were pre-incubated in the absence or presence of ME/Rot (300 $\mathrm{nM} \mathrm{ME} \mathrm{and} 250 \mathrm{nM}$ Rot) for $12 \mathrm{~h}$ in a humidified atmosphere $\left(5 \% \mathrm{CO}_{2}, 37^{\circ} \mathrm{C}\right)$. Mito-TEMPO $(0.1 \mathrm{mM})$ was added to the cell suspensions and the incubation was continued under the same conditions. Aliquots of cell suspensions were collected at different time intervals and subjected to EPR analysis. Control sample contained mito-TEMPO $(0.1 \mathrm{mM})$ in cultured (cell-free) medium. The data are mean $\pm S D$ from four independent experiments.

untreated cells, the intensity of EPR signal decreased significantly, which indicates a conversion of paramagnetic mito-TEMPO to its non-contrast diamagnetic form (hydroxylamine-mito-TEMPOH) due to reduction. The EPR signal decay is a time-dependent process. The signal decreased rapidly within 1-60 min and very slowly within the next $24 \mathrm{~h}$, reaching a plateau. This may be explained by the gradual penetration of mito-TEMPO through the cellular membrane and its subsequent access to the intracellular reducers and oxidizers. Twenty-four hours after the addition of mito-TEMPO to the cell suspension, the intensity of the signal was only $10 \%$ compared to the initial control value $(100 \%)$. The preliminary treatment of cells with the base concentration of ME/Rot for $12 \mathrm{~h}$ influenced the kinetics of EPR signal decay of mito-TEMPO. The signal decreased more slowly, reaching $\sim 30 \%$ intensity at $24 \mathrm{~h}$. This indicates an enhancement of the oxidative capacity of the ME/Rottreated cells in comparison with untreated cells.

Figure 4 shows the dynamics of the EPR signal of mitoTEMPO in normal human lymphocytes, pre-treated with different concentrations of ME/Rot. The signal decreased more slowly and to a lower level with increasing concentration of ME/Rot. At very high concentrations of $\mathrm{ME} / \mathrm{Rot}$ (10- and 20-fold base concentration), the EPR signal was very similar to that of the control level: $80 \%$ (for ME/Rot-treated cells) versus $100 \%$ intensity (for the control), while at lower concentrations (2- and 5-fold base concentration), it decreased significantly, to about $35 \%$ and $60 \%$ intensity, respectively. These data indicate that the oxidative capacity of the cells increased with increasing concentration of ME/Rot.

As mentioned above, our experimental model is based on impairment of mitochondrial respiration by ME/Rot, which is accompanied by overproduction of superoxide (18-20). Thus, in order to verify the EPR data, we used a conventional DHE assay to evaluate the level of superoxide in cells treated with different concentrations of ME/Rot. Figure 5A indicates that the level of superoxide increased with increasing concentration of ME/Rot in the cell suspension. A very good positive correlation between the intensity of EPR signal of mito-TEMPO in the cells and the intracellular level of superoxide, analyzed by DHE, was found (Figure 5B).

Superoxide has a pre-eminent role in biology and pathophysiology because it is formed by many mammalian enzymes, has a key role in cell signaling regulation, and serves as a progenitor for the formation of many other ROS, including hydrogen peroxide, peroxynitrite, and lipid 


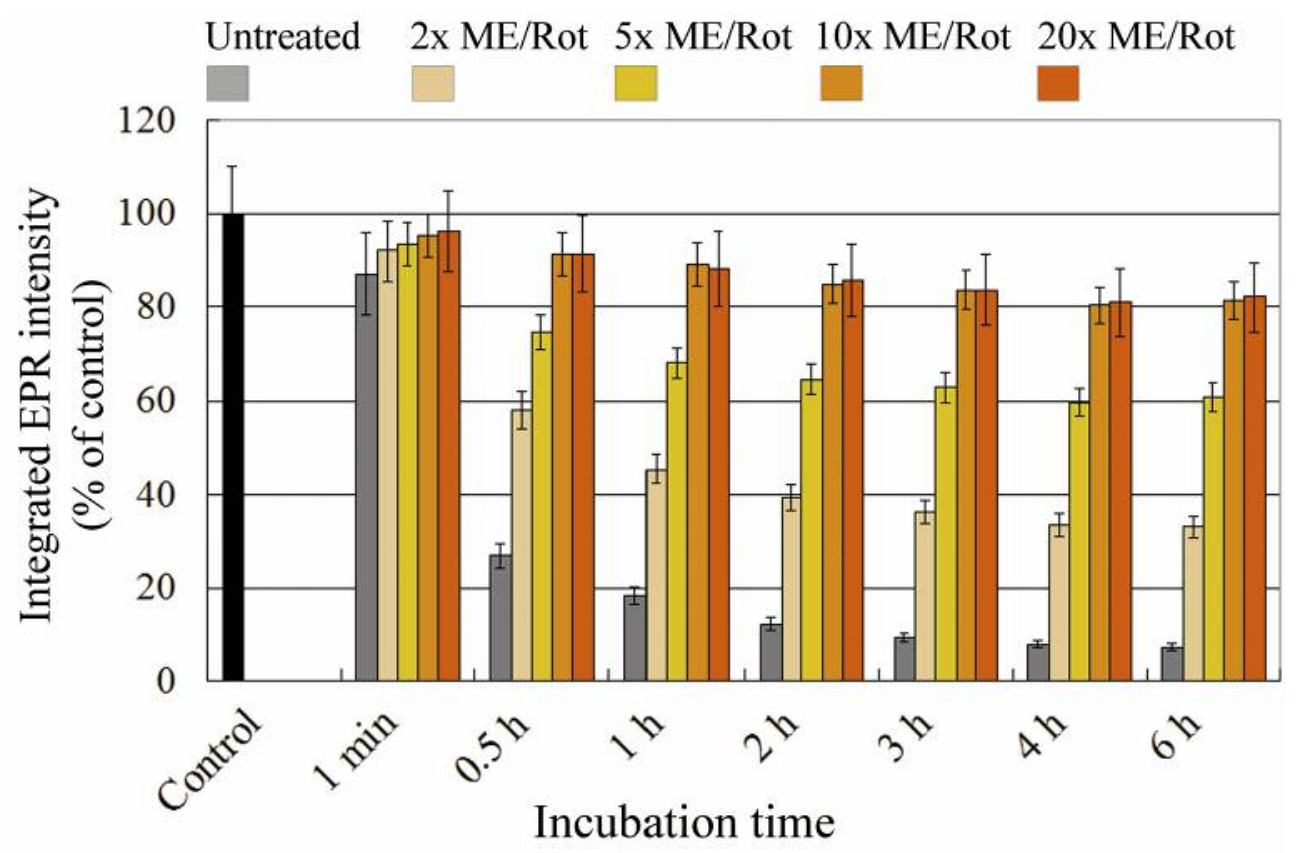

Figure 4. Dynamics of electron-paramagnetic resonance (EPR) signal of (2-(2,2,6,6-tetramethylpiperidin-1-oxyl-4-ylamino)-2oxoethyl)triphenylphosphonium chloride (mito-TEMPO) in normal human lymphocytes, treated with different concentrations of 2-methoxy-estradiol $(M E) /$ rotenone $(R o t)$. Cells $\left(1 \times 10^{6}\right.$ cells $\left./ \mathrm{ml}\right)$ were pre-incubated in the absence or presence of 2-, 5-, 10-, and 20-fold base concentration of ME/Rot $(=300 \mathrm{nM} \mathrm{ME}$ and $250 \mathrm{nM} \mathrm{Rot})$ for $12 \mathrm{~h}$ in a humidified atmosphere $\left(5 \% \mathrm{CO}_{2}, 37^{\circ} \mathrm{C}\right)$. Mito-TEMPO (0.1 $\left.\mathrm{mM}\right)$ was added to the cell suspensions and the incubation was continued under the same conditions. Aliquots of cell suspensions were collected at different time intervals and subjected to EPR analysis. Control sample contained mito-TEMPO $(0.1 \mathrm{mM})$ in cultured (cell-free) medium. The data are mean $\pm S D$ from four independent experiments.

A

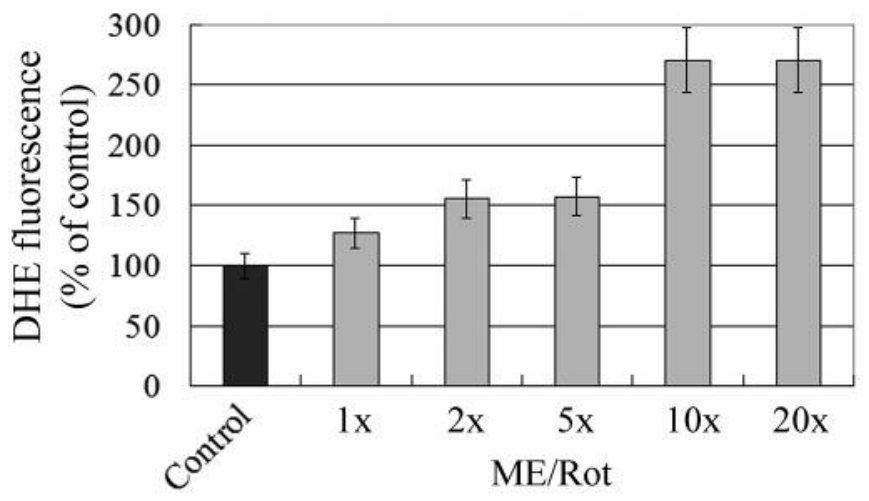

B

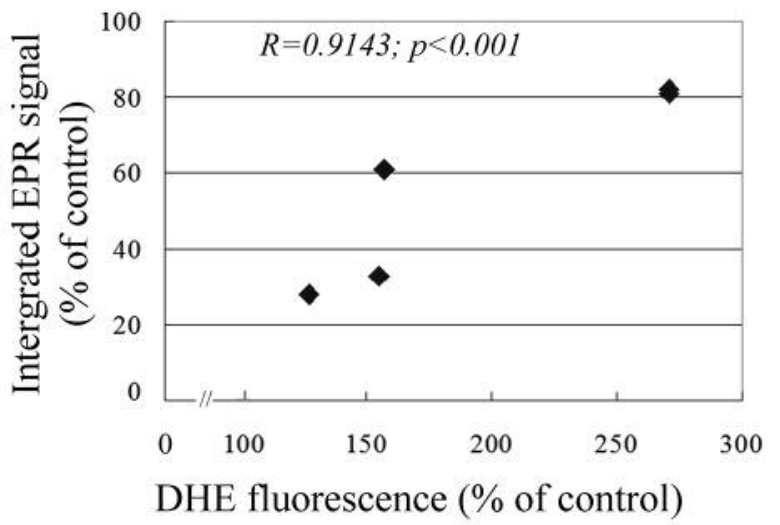

Figure 5. Level of superoxide (A) and correlation between electron-paramagnetic resonance (EPR) intensity of (2-(2,2,6,6-tetramethylpiperidin-1oxyl-4-ylamino)-2-oxoethyl)triphenylphosphonium chloride (mito-TEMPO) and superoxide level $(B)$ in normal human lymphocytes treated with different concentrations of 2-methoxy-estradiol (ME)/rotenone (Rot). A: Cells $\left(1 \times 10^{6} \mathrm{cells} / \mathrm{ml}\right)$ were pre-incubated in the absence (control) or presence of 1-, 2-, 5-, 10-, and 20-fold base concentration of ME/Rot (=300 nM ME and $250 \mathrm{nM}$ Rot) for $12 \mathrm{~h}$ in a humidified atmosphere (5\% $\mathrm{CO}_{2}, 37^{\circ} \mathrm{C}$ ). The level of superoxide was analyzed by dihydroethidium (DHE) test. DHE fluorescence in the control was considered $100 \%$. The data are the mean $\pm S D$ from three independent experiments. B: Cells were treated with different concentrations of ME/Rot (1-, 2-, 5-, 10-, and 20-fold) and the EPR signal intensity was measured $6 \mathrm{~h}$ after addition of mito-TEMPO $(0.1 \mathrm{mM})$ to the cell suspensions, when the kinetic curves reached a plateau. $R$ : Correlation coefficient. 
peroxides (1-3, 5). Moreover, overproduction of superoxide is associated with a variety of pathologies and it is a promising molecular marker for their diagnosis, as well as a target for therapy (1-3).

Our study demonstrated that cell-penetrating paramagnetic spin-probes, such a mito-TEMPO, are valuable tools for EPR imaging of superoxide level in isolated live cells, as well as for EPR imaging of mitochondrial dysfunction and metabolic activity accompanied by superoxide imbalance. This spinprobe is characterized by redox cycle, allowing detection of the balance between intracellular oxidizers and reducers (Figure 1). Therefore, mito-TEMPO is a useful tool for EPR imaging of intracellular redox status in vitro and in vivo.

Recently, Dikalov and Harrison described an excellent overview of the methods for the detection of mitochondrial and cellular ROS (5). Here, we would like to focus only on EPR analysis. One of the earliest methods for detection of superoxide using EPR spectroscopy was spin trapping with 5,5-dimethyl-1-pyrroline-N-oxide (DMPO) (23). It is important to distinguish between spin-traps and spin-probes (5). Spin-traps form covalent bonds with ROS, while spinprobes (such as mito-TEMPO) are oxidized by ROS without being bound (24). DMPO and other similar nitrone spin-traps are very useful in studies of isolated biomacromolecules (e.g. enzymes) and in pure chemical systems. However, they react with superoxide at very slow rate constants and it is difficult to use them for detection of superoxide in biological systems due to competition with SOD and intracellular reducers (such as ascorbate) (25).

Another nitrone spin-trap, 5-diethoxyphosphoryl-5methyl-1-pyrroline N-oxide (DEPMPO), has been conjugated with a triphenylphosphonium group, which allows a selective penetration into mitochondria (26). Unfortunately, the use of this spin-trap, called mitoDEPMPO, is limited by its slow rate constant for reaction with superoxide, potential toxicity and non-specific effects, and its use in biological systems is therefore unlikely $(5,28$, 29). Mito-DEPMPO has to be used at high concentrations (50 mM), which may cause inhibition of mitochondrial respiration due to accumulation of large amounts of lipophilic cations in the mitochondrial matrix and disruption of mitochondrial potential $(5,27)$.

Dikalov et al. reported that cyclic hydroxylamines can be used for measurement of superoxide in cultured cells, tissues, and in vivo (30-32). These diamagnetic molecules are oxidized by superoxide and other ROS to form paramagnetic stable EPR-detectable nitroxide radicals with life-times of several hours in cell culture $(5,18,30-32)$. Cyclic hydroxylamines react with superoxide much more rapidly then nitrone spintraps, which enhances the efficiency for detection of intracellular superoxide (32). These diamagnetic probes can be used at relatively low concentrations (0.05-1 $\mathrm{mM})$, minimizing potential toxic side-effects in biological systems.
In this context, our study demonstrated that paramagnetic spin-probes such as mito-TEMPO are also a promising choice for development of EPR methodologies for detection of superoxide, as well as for evaluation of redox status in live cells and tissues.

\section{Acknowledgements}

The study was partially supported by the Center of Innovation Program from the Japan Science and Technology Agency and by the Diversity Grant, NIRS-Chiba University, Japan (granted to R.A.). The Authors thank Dr. Ken Ichiro Matsumoto (from QST/NIRS) for the possibility to use EPR spectrometer in his lab.

\section{References}

1 Trachootham D, Alexander $\mathbf{J}$ and Huang P: Targeting cancer cells by ROS-mediated mechanisms: a radical therapeutic approach? Nat Rev Drug Discov 8: 579-581, 2009.

2 Pastore A, Petrillo S, Piermarini E and Piemonte F: Systemic redox biomarkers in neurodegenerative diseases. Curr Drug Metabol 16: 46-70, 2015.

3 Rimessi A, Previati M, Nigro F, Wieckowski MR and Pinton P: Mitochondrial reactive oxygen species and inflammation: Molecular mechanisms, diseases and promising therapies. Int $\mathbf{J}$ Biochem Cell Biol 2016, pii: S1357-2725(16)30155-8.

4 Kalyanaraman B, Darley-Usmar V, Davies KJ, Dennery PA, Forman HJ, Grisham MB, Mann GE, Moore K, Roberts LJ and Ischiropoulos $\mathrm{H}$ : Measuring reactive oxygen and nitrogen species with fluorescent probes: challenges and limitations. Free Radic Biol Med 52: 1-6, 2012.

5 Dikalov SI and Harrison DG: Methods for detection of mitochondrial and cellular reactive oxygen species. Antioxid Redox Signal 20: 372-2, 2014.

6 Zhelev Z, Aoki I, Gadjeva V, Nikolova B, Bakalova R and Saga $\mathrm{T}$ : Tissue redox activity as a sensing plarform for imaging of cancer based on nitroxide redox cycle. Eur J Cancer 49: 14671478, 2013.

7 Hyodo F, Murugesan R, Matsumoto K, Hyodo E, Subramanian S, Mitchell JB and Krishna MC: Monitoring redox-sensitive paramagnetic contrast agents by EPRI, ONRI and MRI. J Magn Res 190: 105-112, 2008.

8 Hyodo F, Matsumoto K, Matsumoto A, Mitchell JB and Krishna MC: Probing the intracellular redox status of tumors with magnetic resonance imaging and redox-sensitive contrast agents. Cancer Res 66: 9921-9928, 2006.

9 Davis RM, Matsumoto S, Bernardo M, Sowers A, Matsumoto $\mathrm{K}$, Krishna MC and Mitchell JB: Magnetic resonance imaging of organic contrast agents in mice: capturing the whole-body redox landscape. Free Radic Biol Med 50: 459-468, 2011.

10 Matsumoto K, Hyodo F, Matsumoto A, Koretsky AP, Sowers AL, Mitchell JB and Krishna MC: High-resolution mapping of tumour redox status by MRI using nitroxides as redox-sensitive contrast agents. Clin Cancer Res 12: 2355-2362, 2006.

11 Fuchs J, Groth N, Herrling $\mathrm{T}$ and Zimmer G: Electron paramagnetic resonance studies on nitroxide radical 2,2,5,5tetramethyl-4-piperidin-1-oxyl (TEMPO) redox reactions in human skin. Free Radic Biol Med 22: 967-976, 1997. 
12 Batinic-Haberle I, Reboucas JS and Spasijevic I: Superoxide dismutase mimetics: chemistry, pharmacology, and therapeutic potential. Antioxid Redox Signal 13: 877-918, 2010.

13 Mehlhorn RJ: Ascorbate- and dehydroascorbic acid-mediated reduction of free radicals in the human erythrocytes. J Biol Chem 266: 2724-2731, 1991.

14 Bobko AA, Kirilyuk IA, Grigor'ev IA, Zweier JL and Khramtsov VV: Reversible reduction of nitroxides to hydroxylamines: the roles for ascorbate and glutathione. Free Radic Biol Med 42: 404-412, 2007.

15 Matsumoto A, Matsumoto K, Matsumoto S, Hyodo F, Sowers AL, Koscielniak JW, Krishna MC and Mitchell JB: Intracellular hypoxia of tumour tissue estimated by noninvasive EPR oximetry technique using paramagnetic probes. Biol Pharm Bull 34: 142-145, 2011.

16 Goldman S, Merenyi G, Russo A and Samuni A: The role of oxoammonium cation in the SOD-mimic activity of cyclic nitroxides. J Am Chem Soc 125: 789-795, 2003.

17 Zhelev Z, Bakalova R, Aoki I, Lazarova D and Saga T: Imaging of superoxide Generation in the dopaminergic area of the brain in Parkinson's disease, using mito-TEMPO. ACS Chem Neurosci 4: 1439-1445, 2013.

18 Bakalova R, Georgieva E, Ivanova D, Zhelev Z, Aoki I and Saga $\mathrm{T}$ : Magnetic resonance imaging of mitochondrial dysfunction and metabolic activity, accompanied by overproduction of superoxide. ACS Chem Neurosci 6: 1922-1929, 2015.

19 Pelicano H, Feng L, Zhou Y, Carew JS, Hileman EO, Plunkett W, Keating MJ and Huang P: Inhibition of mitochondrial respiration: A novel strategy to enhance drug-induced apoptosis in human leukemia cells by a reactive oxygen species-mediated mechanism. J Biol Chem 278: 37832-37839, 2003.

20 Huang P, Feng L, Oldham EA, Keating MJ and Plunkett W: Superoxide dismutase as a target for the selective killing of cancer cells. Nature 407: 390-395, 200.

21 Budd S, Castilho R and Nicholls D: Mitochondrial membrane potential and hydroethidine-monitored superoxide generation in cultured cerebellar granule cells. FEBS Lett 415: 21-24, 1997.

22 Peshavariya H, Dusting $G$ and Selemidis S: Analysis of dihydroethidium fluorescence for the detection of intracellular and extracellular superoxide produced by NADPH oxidase. Free Radic Res 41: 699-712, 2007.

23 Finkelstein E, Rosen GM and Rauckman EJ: Spin trapping of superoxide and hydroxyl radical: practical aspects. Arch Biochem Biophys 200: 1-16, 1980.
24 Dikalov S, Griendling KK and Harrison DG: Measurement of reactive oxygen species in cardiovascular studies. Hypertension 49: 717-727, 2007.

25 Zhao H, Joseph J, Fales HM, Sokoloski EA, Levine RL, Vasquez-Vivar $\mathrm{J}$ and Kalyanaraman B: Detection and characterization of the product of hydroethidine and intracellular superoxide by HPLC and limitations of fluorescence. Proc Natl Acad Sci USA 102: 5727-5732, 2005.

26 Hardy M, Rockenbauer A, Vasquez-Vivar J, Felix C, Lopez M, Srinivasan S, Avadhani N, Tordo P and Kalyanaraman B: Detection, characterization, and decay kinetics of ROS and thiyl adducts of mito-DEPMPO spin trap. Chem Res Toxicol 20: 1053-1060, 2007.

27 Kurtoglu M and Lampidis TJ: From delocalized lipophilic cations to hypoxia: blocking tumor cell mitochondrial function leads to therapeutic gain with glycolytic inhibitors. Mol Nutr Food Res 53: 68-75, 2009.

28 Keszler A, Kalyanaraman B and Hogg N: Comparative investigation of superoxide trapping by cyclic nitrone spin traps: the use of singular value decomposition and multiple linear regression analysis. Free Radic Biol Med 35: 1149-1157, 2003.

29 Khan N, Wilmot CM, Rosen GM, Demidenko E, Sun J, Joseph J, O'Hara J, Kalyanaraman B and Swartz HM: Spin traps: in vitro toxicity and stability of radical adducts. Free Radic Biol Med 34: 1473-1481, 2003.

30 Dikalov S, Fink B, Skatchkov M and Bassenge E: Comparison of glyceryl trinitrate-induced with pentaerythrityl tetranitrateinduced in vivo formation of superoxide radicals: effect of vitamin C. Free Radic Biol Med 27: 170-76, 1999.

31 Dikalov S, Skatchkov M, Fink B and Bassenge E: Quantification of superoxide radicals and peroxynitrite in vascular cells using oxidation of sterically hindered hydroxylamines and electron spin resonance. Nitric Oxide 1: 423-431, 1997.

32 Dikalov SI, Dikalova AE and Mason RP: Noninvasive diagnostic tool for inflammation-induced oxidative stress using electron-spin resonance spectroscopy and an extracellular cyclic hydroxylamine. Arch Biochem Biophys 402: 218-226, 2002.

Received August 11, 2016

Revised September 3, 2016

Accepted September 12, 2016 